\title{
Intellisỵs
}

7-8 September 2017 | London, UK

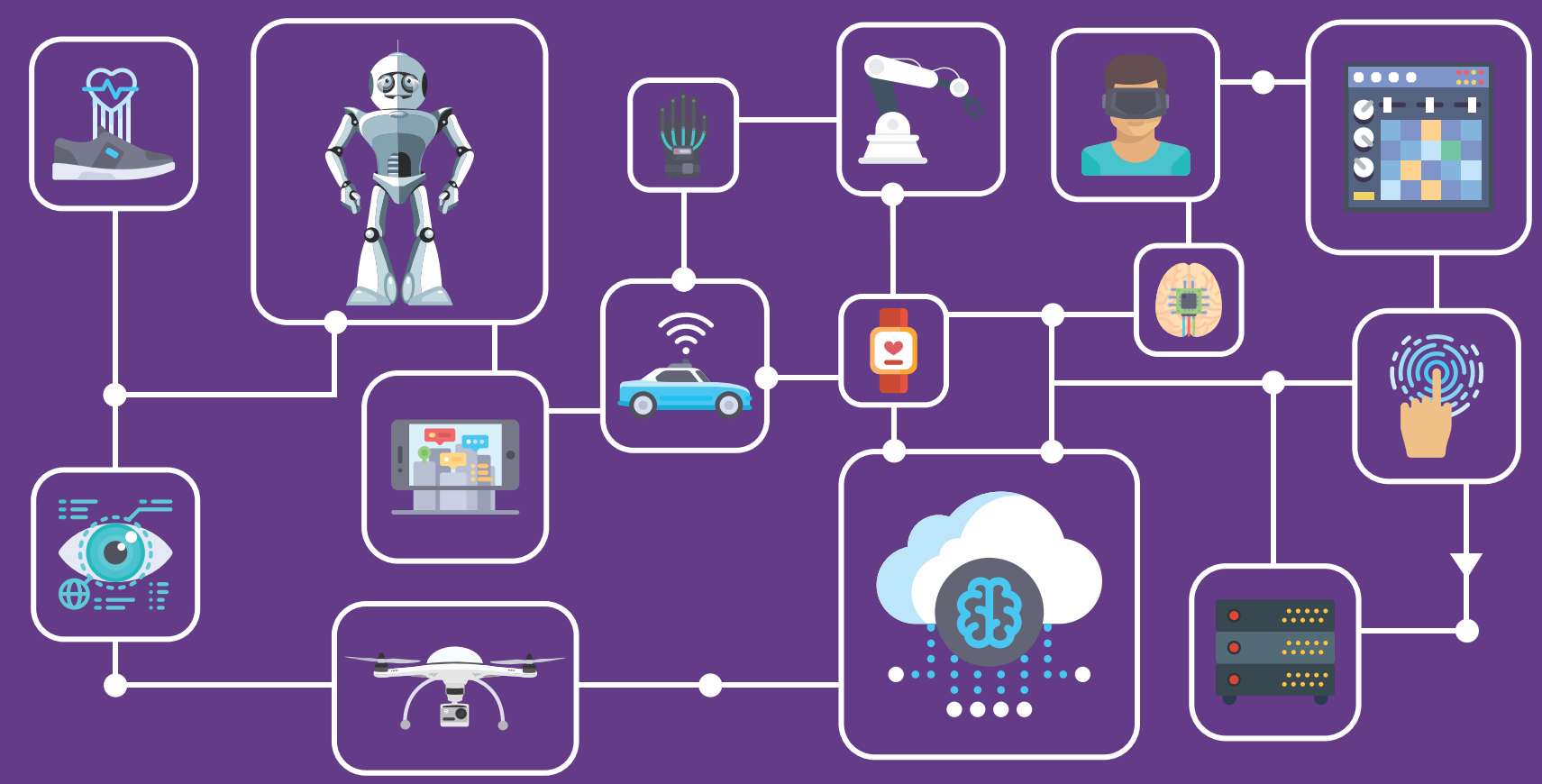

ORGANIZED BY

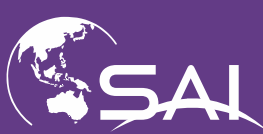

THE SCIENCE AND INFORMATION
TECHNICAL SPONSOR

4. IEEE web: www.saiconference.com/IntelliSys

email: intellisys@saiconference.com

Ð)@SAIConference

f /SAIConference 


\section{CONFERENCE AGENDA}

\section{DAY 1 (7th September)}

8:00 am

Delegates Check-in

8:30 am - 10:30 am KEYNOTE TALKS (Ludgate Suite)

Valentina Salapura, IBM

Frank Wang, University of Kent

Jan Hofmann, Deutsche Telekom AG

10:30 am - 11:00 am POSTER PRESENTATIONS (Cornhill Room)

11:00 am - 12:30 pm PAPER PRESENTATIONS

Session 1: Ambient Intelligence

Session 2: Data Science

Session 3: Machine Learning

Session 4: Human Computer Interaction

12:30 pm - 1:30 pm Lunch (Cornhill Room)

$1: 30 \mathrm{pm}-3: 30 \mathrm{pm}$

\section{PAPER PRESENTATIONS}

Session 5: Ambient Intelligence

Session 6: Data Science

Session 7: Machine Learning

Session 8: Artificial Intelligence

3:30 pm - 3:45 pm

3:45 pm - 5:45 pm

Networking Break (Cornhill Room)

PAPER PRESENTATIONS

Session 9: Image Processing

Session 10: Data Science

Session 11: Machine Learning

Session 12: Artificial Intelligence

6:00 pm - 10:00 pm EVENING LONDON TOUR

(Dinner / Meals are not included)

\section{DAY 2 (8th September)}

\begin{tabular}{|c|c|}
\hline $8: 00 \mathrm{am}$ & Delegates Arrive \\
\hline $8: 30$ am - 10:15 am & $\begin{array}{l}\text { KEYNOTE TALKS (Ludgate Suite) } \\
\text { Paolo Rosso, Technical University of Valencia } \\
\text { Nuria Oliver, Vodafone }\end{array}$ \\
\hline $10: 15 \mathrm{am}-10: 30 \mathrm{am}$ & Networking Break (Cornhill Room) \\
\hline $10: 30 \mathrm{am}-12: 30 \mathrm{pm}$ & $\begin{array}{l}\text { PAPER PRESENTATIONS } \\
\text { Session 13: Intelligent Systems } \\
\text { Session 14: Security } \\
\text { Session 15: Machine Vision } \\
\text { Session 16: Expert Systems }\end{array}$ \\
\hline $12: 30 \mathrm{pm}-1: 30 \mathrm{pm}$ & Lunch (Cornhill Room) \\
\hline $1: 30$ pm - 3:30 pm & $\begin{array}{l}\text { PAPER PRESENTATIONS } \\
\text { Session 17: Intelligent Systems } \\
\text { Session 18: Security } \\
\text { Session 19: Machine Vision } \\
\text { Session 20: Robotics }\end{array}$ \\
\hline $3: 30$ pm - 3:45 pm & Networking Break (Cornhill Room) \\
\hline $3: 45 p m-5: 15 p m$ & $\begin{array}{l}\text { PAPER PRESENTATIONS } \\
\text { Session 21: Ambient Intelligence } \\
\text { Session 22: Neural Networks } \\
\text { Session 23: Natural language processing } \\
\text { Session 24: Robotics }\end{array}$ \\
\hline
\end{tabular}

5:15 pm - 5:30 pm Conference Closing (Ludgate Suite) 


\section{KEYNOTE SPEAKERS}

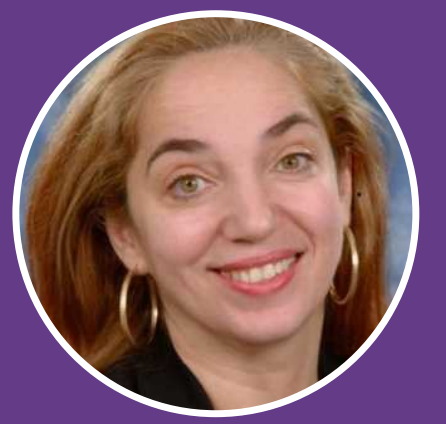

Valentina Salapura

IBM T.J. Watson Research Center

7th September | 8:45 am - 9:30 am

\section{Jan Hofmann}

Deutsche Telekom AG

7th September | 10:00 am - 10:30 am

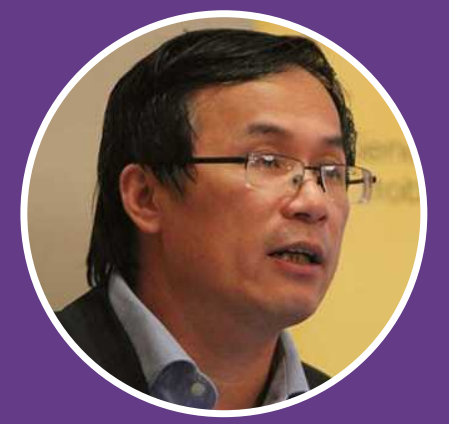

Frank Wang

University of Kent \& Chair IEEE UKRI

7th September | 9:30 am - 10:00 am

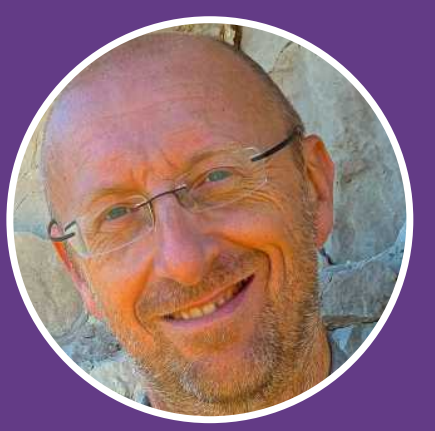

\section{Paolo Rosso}

Technical University of Valencia, Spain

8th September | 8:30 am - 9:00 am

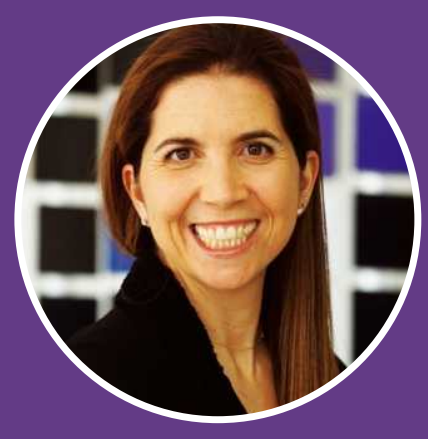

Nuria Oliver

Vodafone

8th September | 9:00 am - 9:30 am 


\section{7th September (10:30 am - 11:00 am) \\ POSTER PRESENTATIONS \\ Session Chair: Kohei Arai}

171 - A Tourist Advisor based on a Question Answering System

(Presenter: Gianpaolo Ghiani, Italy)

286 - Finding Bottlenecks: Predicting Student Attrition with Unsupervised Classifier (Presenter: Bruce Shapiro, United States)

409 - Intra-Class Complex Object Shape Representation Towards High Resolution (Presenter: Xinhan Di, Ireland)

193 - Utilizing High-Performance Embedded Computing, Agile Condor, for Intelligent Processing

(Presenter: Mark Barnell, United States)

39 - Comparison of Adaptive Algorithms for Single-Trial Event-Related Potentials Extraction (Presenter: Moncef BENKHERRAT, France)

346 - The Human on the Loop

(Presenter: David Johnson, United States)

6 - Cluster Restarted DM: New Algorithm for Global Optimisation (Presenter: Marek Dlapa, Czech Republic)

336 - Multi-Dimensional I-Vector Closed Set Speaker Identification based on an Extreme Learning Machine with and without Fusion Technologies

(Presenter: Musab Al-kaltakchi, United Kingdom)

183 - Visualizing Positive and Negative Affordances in Infancy using Mobile Augmented Reality

(Presenter: Miho Nishizaki, Japan)

\section{EVENING LONDON TOUR ON 7th September!}

6:00 pm - 8:00 pm: Panoramic coach tour seeing Royal Albert Hall, Natural History Museum, Harrods, Hyde Park Corner, Big Ben and the Houses of Parliament, Whitehall, Trafalgar Sq, The Strand

8:00 pm - 10:00 pm: Stopover at Covent Garden (has nice dinner options/ cuisines - delegates to pay on their own)

$10 \mathrm{pm}$ : Disembark and Transfer back to the conference venue

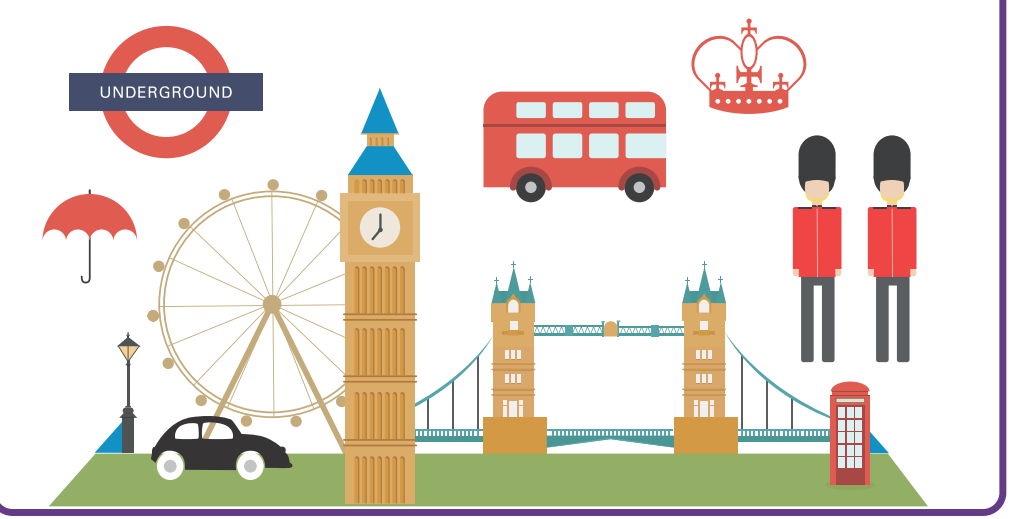


7th September (11:00 am - 12:30 pm)

PAPER PRESENTATIONS

\begin{tabular}{|c|c|c|c|}
\hline $\begin{array}{l}\text { Session 1: Ambient Intelligence } \\
\text { Session Chair: Michael Farmer } \\
\text { (Ludgate Suite) }\end{array}$ & $\begin{array}{l}\text { Session 2: Data Science } \\
\text { Session Chair: Parisa Naraei } \\
\text { (Walbrook Suite) }\end{array}$ & $\begin{array}{l}\text { Session 3: Machine Learning } \\
\text { Session Chair: Yaxin Bi } \\
\text { (Fleet Suite) }\end{array}$ & $\begin{array}{l}\text { Session 4: Human Computer Interaction } \\
\text { Session Chair: Mark Keane } \\
\text { (Bishopsgate Suite) }\end{array}$ \\
\hline $\begin{array}{l}153 \text { - Towards Adaptive Control in Smart } \\
\text { Homes (Presenter: Zelin Wang, United } \\
\text { Kingdom) } \\
94 \text { - Results from Investigating Powered } \\
\text { Wheelchair Users Learning to Drive with } \\
\text { Varying Levels of Sensor Support (Presenter: } \\
\text { David Sanders, United Kingdom) } \\
154 \text { - ML-IDS: A Machine Learning Approach to } \\
\text { Detect Wormhole Attacks in Internet of Things } \\
\text { (Presenter: Prachi Shukla, United States) } \\
24 \text { - A Smart Lighting System using Wireless } \\
\text { Sensor Actuator Network (Presenter: Sanjib } \\
\text { Panda, Singapore) } \\
263 \text { - Implementation of Evolutionary } \\
\text { Algorithms in Vehicular Ad-Hoc Network for } \\
\text { Cluster Optimization (Presenter: Muhammad } \\
\text { Fahad, Pakistan) }\end{array}$ & $\begin{array}{l}176 \text { - Features Extracted from APPES to Enable } \\
\text { the Categorization of Heavy-Duty Vehicle } \\
\text { Drivers (Presenter: Iulian Carpatorea, Sweden) } \\
297 \text { - Boolean Prediction of Final Grades based } \\
\text { on Weekly and Cumulative Activities } \\
\text { (Presenter: Mohammad Majid al-Rifaie, United } \\
\text { Kingdom) } \\
189 \text { - A Data Reduction Method based on } \\
\text { Indiscernibility and Rough Entropy for } \\
\text { Uncertain Systems (Presenter: Udayan Ghose, } \\
\text { India) } \\
294 \text { - A Framework for Pattern Based Melody } \\
\text { Matching for Content Based Music Information } \\
\text { Retrieval (Presenter: Vikram Dara, India) } \\
320 \text { - Presence Analytics: Detecting Classroom- } \\
\text { based Social Patterns using WLAN Traces } \\
\text { (Presenter: Muawya Eldaw, United Kingdom) }\end{array}$ & $\begin{array}{l}143 \text { - Real-Time Usage Forecasting for Bike- } \\
\text { Sharing Systems (Presenter: Simon Ruffieux, } \\
\text { Switzerland) } \\
113 \text { - Implementing a Cross-Curricular Digital } \\
\text { Project into a PGCE Computer Science Initial } \\
\text { Teacher Education Course (Presenter: Carl } \\
\text { Wilkinson, United Kingdom) } \\
279 \text { - Classification of Imbalanced Data in E- } \\
\text { Commerce (Presenter: Liliya Besaleva, United } \\
\text { States) } \\
60 \text { - Early Warning Modeling and Application } \\
\text { based on Analytic Hierarchy Process Integrated } \\
\text { Extreme Learning Machine (Presenter: } \\
\text { YongMing Han, China) } \\
251 \text { - Automated Farming Prediction (Talha } \\
\text { Siddique) }\end{array}$ & $\begin{array}{l}61 \text { - Rule-Based System to Assist a Powered } \\
\text { Wheelchair Driver (Presenter: Giles } \\
\text { Tewkesbury, United Kingdom) } \\
140 \text { - Pervasive Audio Playback in Cyber- } \\
\text { Physical Environments (Presenter: Yannick } \\
\text { Koerber, Germany) } \\
130 \text { - Minimalist Design: An Optimized } \\
\text { Solution for Intelligent Interactive Infotainment } \\
\text { Systems (Presenter: Ashraf Gaffar, United } \\
\text { States) } \\
32 \text { - New Method for Optimization of Static } \\
\text { Hand Gesture Recognition (Presenter: Alaa } \\
\text { Omran, Iraq) } \\
85 \text { - Contagion: Optimizing Foodborne } \\
\text { Outbreak Analysis with Automatic Suggestions } \\
\text { (Presenter: Dan Guo, United States) }\end{array}$ \\
\hline
\end{tabular}




\section{7th September (1:30 pm - 3:30 pm)}

\section{PAPER PRESENTATIONS}

\begin{tabular}{|c|c|c|c|}
\hline $\begin{array}{l}\text { Session 5: Ambient Intelligence } \\
\text { Session Chair: Yaxin Bi } \\
\text { (Ludgate Suite) }\end{array}$ & $\begin{array}{l}\text { Session 6: Data Science } \\
\text { Session Chair: Parisa Naraei } \\
\text { (Walbrook Suite) }\end{array}$ & $\begin{array}{l}\text { Session 7: Machine Learning } \\
\text { Session Chair: Carl Wilkinson } \\
\text { (Fleet Suite) }\end{array}$ & $\begin{array}{l}\text { Session 8: Artificial Intelligence } \\
\text { Session Chair: Kohei Arai } \\
\text { (Bishopsgate Suite) }\end{array}$ \\
\hline $\begin{array}{l}303 \text { - Context Classification in Energy Resource } \\
\text { Management of Residential Buildings using } \\
\text { Artificial Neural Network (Presenter: Carlos } \\
\text { Ramos, Portugal) } \\
148 \text { - Femtocell Deployment Plan: Moving } \\
\text { Indoors (Presenter: Abdullah Alotaibi, United } \\
\text { Kingdom) } \\
115 \text { - A Single Chip System for Sensor Data } \\
\text { Fusion based on a Drift-Diffusion Model } \\
\text { (Presenter: Shufan Yang, United Kingdom) } \\
313 \text { - Towards Stream-based Reasoning and } \\
\text { Machine Learning for loT Applications } \\
\text { (Presenter: Markus Endler, Brazil) } \\
367 \text { - A Simple Integrated Smart Green Home } \\
\text { Design (Presenter: Bader Alyoubi, Ibraheem } \\
\text { Alharbi, Ashraf Badawood, Saudi Arabia) } \\
388 \text { - The Role of Voice Service Technologies in } \\
\text { Creating the Next Generation Outpatient Data } \\
\text { Driven Electronic Health Record (EHR) } \\
\text { (Presenter: Chandrasekar Vuppalapati, United } \\
\text { States) } \\
265 \text { - Traffic Game in Smart City for Traffic } \\
\text { Management (Presenter: Tanghsien Chang, } \\
\text { China) }\end{array}$ & $\begin{array}{l}237 \text { - Discovering News Events that Move } \\
\text { Markets (Presenter: Mark Keane, Ireland) } \\
135 \text { - An Intelligent System for Forex Trading: } \\
\text { Hybrid ANN with GARCH and Intrinsic Mode } \\
\text { Functions (Presenter: Hasanthi Pathberiya, Sri } \\
\text { Lanka) } \\
401 \text { - Identifying Accurate Classifier Models for } \\
\text { a Text-based MERS-Cov Dataset (Presenter: } \\
\text { Norah Almansour, Saudi Arabia) } \\
121 \text { - XML-Relational Mapping using } \\
\text { Production Rule System (Presenter: Andrey } \\
\text { Lyamin, Russia) } \\
125 \text { - LUCID: Author Name Disambiguation } \\
\text { using Graph Structural Clustering (Presenter: } \\
\text { Sohail Asghar, Pakistan) } \\
90 \text { - Knowledge Extraction from Software } \\
\text { Engineering Repositories (Presenter: N V } \\
\text { Ramana Rao Gundu, India) } \\
211 \text { - A Modified Penalty Function in Fuzzy } \\
\text { Clustering Algorithm (Presenter: Md Shahjahan, } \\
\text { Bangladesh) }\end{array}$ & $\begin{array}{l}180 \text { - Machine Learning and Deep Neural } \\
\text { Network - Artificial Intelligence Core for Lab } \\
\text { and Real-World Test and Validation for ADAS } \\
\text { and Autonomous Vehicles (Presenter: Harsha } \\
\text { Jakkanahalli Vishnukumar, Björn Butting, } \\
\text { Germany) } \\
118 \text { - Exploiting Action Categories in Learning } \\
\text { Complex Games (Presenter: Mihai Sorin Dobre, } \\
\text { United Kingdom) } \\
84 \text { - Evaluating Cross Domain Sentiment } \\
\text { Analysis using Supervised Machine Learning } \\
\text { Techniques (Presenter: Azwa Abdul Aziz, United } \\
\text { Kingdom) } \\
188 \text { - Tag based Models for Arabic Text } \\
\text { Compression (Presenter: William Teahan, } \\
\text { United Kingdom) } \\
290 \text { - Predicting All Star Player in the National } \\
\text { Basketball Association using Random Forest } \\
\text { (Presenter: Ala'a El-Nabawy, Egypt) } \\
28 \text { - Predicting Lung Cancer Survivability using } \\
\text { Ensemble Learning Methods (Presenter: Reza } \\
\text { Javidan, Iran) } \\
277 \text { - An Expert System for Diabetes Prediction } \\
\text { using Auto Tuned Multi-Layer Perceptron } \\
\text { (Presenter: Maham Jahangir, Pakistan) }\end{array}$ & $\begin{array}{l}156 \text { - A Social Influence Model for Exploring } \\
\text { Double Subjectivity through News Frames in } \\
\text { Online News (Presenter: Loretta Cheeks, United } \\
\text { States) } \\
380 \text { - Unraveling an Elusive Future: Social } \\
\text { Inequality and Social Responsibility in the Tech } \\
\text { Industry (Presenter: Soraya Cardenas-Conte, } \\
\text { United States) } \\
35 \text { - Information Compression, Multiple } \\
\text { Alignment, and Intelligence (Presenter: Gerry } \\
\text { Wolff, United Kingdom) } \\
158 \text { - A New Min-Transitive Fuzzy Left-Relation } \\
\text { on Intervals for a Different Class of Problems } \\
\text { (Presenter: Sukhamay Kundu, United States) } \\
345 \text { - Developing Video Games with } \\
\text { Elementary Adaptive Artificial Intelligence in } \\
\text { Unity (Presenter: Apostolos Meliones, Greece) } \\
403 \text { - Towards a New Interpretation of } \\
\text { Separable (Presenter: Tapabrata Ghosh, United } \\
\text { States) } \\
314 \text { - Sequential Evidence Accumulation via } \\
\text { Integrating Dempster-Shafer Reasoning and } \\
\text { Estimation Theory (Presenter: Michael Farmer, } \\
\text { United States) }\end{array}$ \\
\hline
\end{tabular}




\section{7th September (3:45 pm - 5:45 pm)}

\section{PAPER PRESENTATIONS}

\begin{tabular}{|c|c|c|c|}
\hline $\begin{array}{l}\text { Session 9: Image Processing } \\
\text { Session Chair: Vassilis S. Kodogiannis } \\
\text { (Ludgate Suite) }\end{array}$ & $\begin{array}{l}\text { Session 10: Data Science } \\
\text { Session Chair: Mark Keane } \\
\text { (Walbrook Suite) }\end{array}$ & $\begin{array}{l}\text { Session 11: Machine Learning } \\
\text { Session Chair: Yaxin Bi } \\
\text { (Fleet Suite) }\end{array}$ & $\begin{array}{l}\text { Session 12: Artificial Intelligence } \\
\text { Session Chair: Kohei Arai } \\
\text { (Bishopsgate Suite) }\end{array}$ \\
\hline $\begin{array}{l}150 \text { - Stacking Approach for CNN Transfer } \\
\text { Learning Ensemble for Remote Sensing } \\
\text { Imagery (Presenter: Timothy Andersen, United } \\
\text { States) } \\
202 \text { - A Review on Image Segmentation based } \\
\text { on Multi-Agent Systems (Presenter: Maroua } \\
\text { Amahrir, Morocco) } \\
181 \text { - SQbSN: JPEG2000 Scalar Quantizer } \\
\text { Implemented by Means a Statistical } \\
\text { Normalization (Presenter: Jesus Jaime Moreno- } \\
\text { Escobar, Mexico) } \\
372 \text { - Face Recognition under Varying } \\
\text { Illumination based on Homomorphic Filter and } \\
\text { Local Binary Patterns (Presenter: Shorouq } \\
\text { Alotaibi, Saudi Arabia) } \\
123 \text { - Recent Advances in Image Processing } \\
\text { Techniques for Automated Harvesting } \\
\text { Purposes: A Review (Presenter: Manuel Reis, } \\
\text { Portugal) } \\
285 \text { - FPGA based Image De-Hazing } \\
\text { Architecture for Real Time Applications } \\
\text { (Presenter: Gulistan Raja, Pakistan) } \\
174 \text { - Automatic Tracking of Cervical Spine } \\
\text { using Fluoroscopic Sequences (Presenter: Ali } \\
\text { Hassan, Pakistan) }\end{array}$ & $\begin{array}{l}347 \text { - Dataset Designing of Software } \\
\text { Architectures Styles for Analysis through Data } \\
\text { Mining Clustering Algorithms (Presenter: } \\
\text { Qadeem Khan, Pakistan) } \\
149 \text { - From Data Exploration to Semantic Model } \\
\text { of Customer (Presenter: Jerzy Korczak, Poland) } \\
325 \text { - Toward Learning Intracranial } \\
\text { Hypertension through Physiological Features } \\
\text { (Presenter: Parisa Naraei, Canada) } \\
147 \text { - Dependency Networks Extractions from } \\
\text { Textual Sources - A Case study in Criminology } \\
\text { (Presenter: Marcello Trovati, United Kingdom) } \\
37 \text { - Partially Squeezing the Resources of the } \\
\text { Web of Data towards Applications (Presenter: } \\
\text { Ana Garcia-Serrano, Spain) } \\
253 \text { - Improving Open-Domain Event Schema } \\
\text { Discovery with Casual English Normalization } \\
\text { for Noisy Text (Presenter: Assia Mezhar, } \\
\text { Morocco) } \\
15 \text { - The Cyber Teamwork System based on } \\
\text { Cloud to Maximize the Teamwork Capability } \\
\text { (Presenter: Narungsun Wilaisakoolyong, } \\
\text { Thailand) } \\
318 \text { - Towards Intelligent Open Data Platforms } \\
\text { (Presenter: Oladipupo Sennaike, Nigeria) }\end{array}$ & $\begin{array}{l}65 \text { - Generalized Relevance Vector Machine } \\
\text { (Presenter: Sam Kwong, Yuheng Jia, Hong } \\
\text { Kong) } \\
81 \text { - How Efficient is Twitter: Predicting } 2012 \\
\text { U.S. Presidential Elections using Support Vector } \\
\text { Machine via Twitter and Comparing Against } \\
\text { lowa Electronic Markets (Presenter: Abbas } \\
\text { Attarwala, Canada) } \\
19 \text { - Intelligent Search with Deep Learning } \\
\text { Clusters (Presenter: Will Serrano, United } \\
\text { Kingdom) } \\
200 \text { - Maximum Distance Minimum Error } \\
\text { (MDME): A Non-Parametric Approach to } \\
\text { Feature Selection for Image-based High } \\
\text { Content Screening Data (Presenter: Robert } \\
\text { Trevino, United States) } \\
192 \text { - Bio-Inspired Meta-Learning for Active } \\
\text { Exploration During Non-Stationary } \\
\text { Multi-Armed Bandit Tasks (Presenter: Georgios } \\
\text { Velentzas, Greece) } \\
173 \text { - Data Mining and Model-Predictive } \\
\text { Approach for Blast Furnace Thermal Control } \\
\text { (Presenter: Dmitry Shnayder, Russia) } \\
276 \text { - MCS: Multiple Classifier System to Predict } \\
\text { the Churners in the Telecom Industry } \\
\text { (Presenter: Mehreen Ahmed, Pakistan) }\end{array}$ & $\begin{array}{l}161 \text { - Simulation-Optimisation of Multi-Product, } \\
\text { Multi-Period Consumer Supply Network using } \\
\text { Genetic Algorithms (Presenter: Zeinab } \\
\text { Hajiabaolhasani, Australia) } \\
310 \text { - Decision Support in Condition-based } \\
\text { Maintenance of a Fleet of Cyber-Physical } \\
\text { Systems (Presenter: John Mbuli, France) } \\
382 \text { - A Reconfigurable Architecture for Real- } \\
\text { Time Digital Simulation of Neurons (Presenter: } \\
\text { Jonathan Graham-Harper-Cater, United } \\
\text { Kingdom) } \\
412 \text { - Amplifying Prediction Accuracy using } \\
\text { Swarm A.I. (Presenter: Louis Rosenberg, United } \\
\text { States) } \\
231 \text { - Algorithms and Approaches to } \\
\text { Mathematical Modeling of Conflict in a } \\
\text { Complex Social System (Presenter: Alexandr } \\
\text { Petukhov, Russia) } \\
381 \text { - Hybridization of Moth Flame } \\
\text { Optimization and Gravitational Search } \\
\text { Algorithm and its Application to Detection of } \\
\text { Food Quality (Presenter: Anirudh Bhutani, } \\
\text { India) } \\
337 \text { - Discriminant Learning for Hybrid } \\
\text { HMM/MLP Speech Recognition System using a } \\
\text { Fuzzy Genetic Clustering (Presenter: Lilia Lazli, } \\
\text { Canada) }\end{array}$ \\
\hline
\end{tabular}


8th September (10:30 am - 12:30 pm)

PAPER PRESENTATIONS

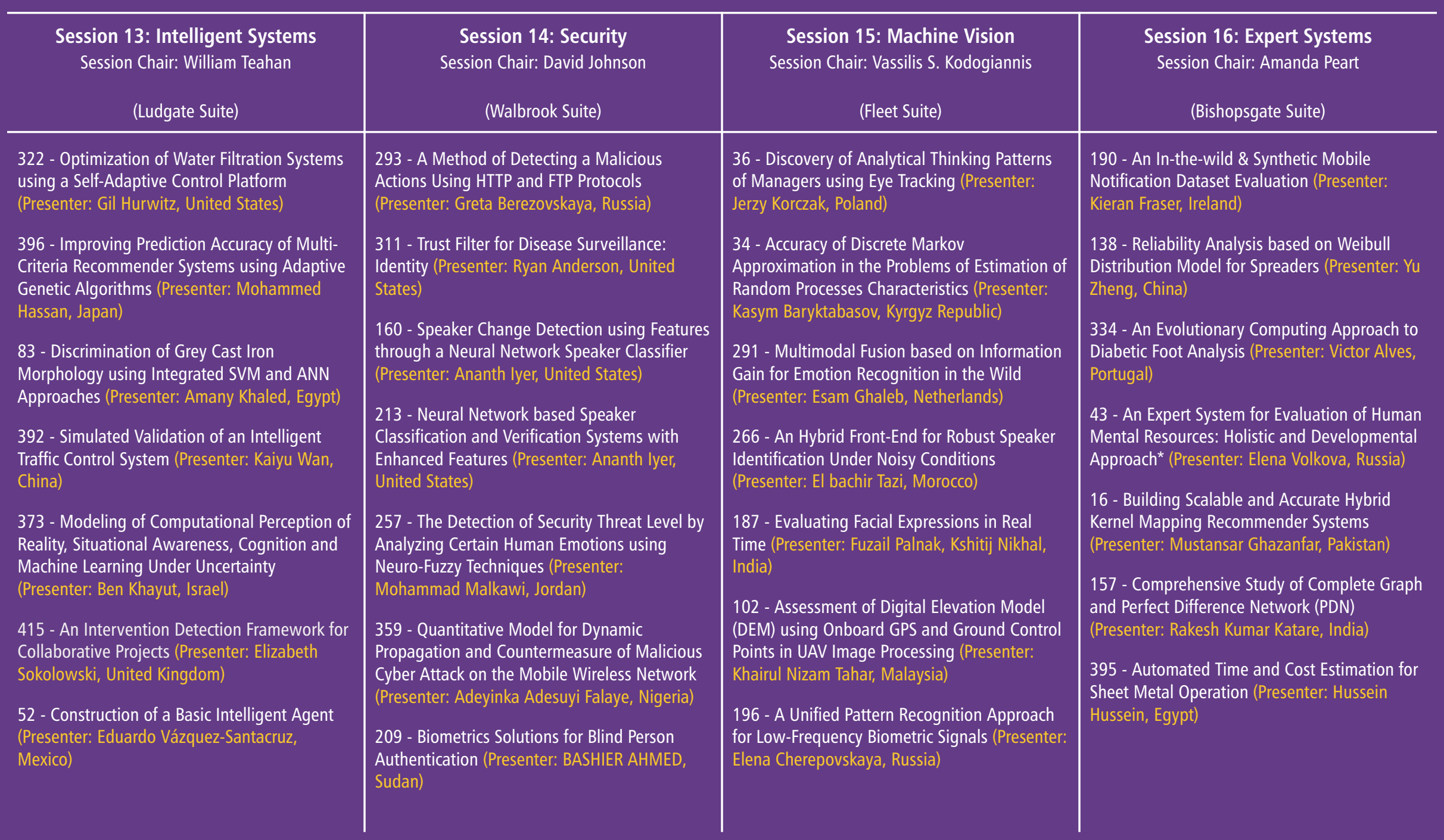


8th September (1:30 pm - 3:30 pm)

PAPER PRESENTATIONS

\begin{tabular}{|c|c|c|c|}
\hline $\begin{array}{l}\text { Session 17: Intelligent Systems } \\
\text { Session Chair: William Teahan } \\
\text { (Ludgate Suite) }\end{array}$ & $\begin{array}{l}\text { Session 18: Security } \\
\text { Session Chair: David Johnson } \\
\text { (Walbrook Suite) }\end{array}$ & $\begin{array}{l}\text { Session 19: Machine Vision } \\
\text { Session Chair: Yaxin Bi } \\
\text { (Fleet Suite) }\end{array}$ & $\begin{array}{l}\text { Session 20: Robotics } \\
\text { Session Chair: Kohei Arai } \\
\text { (Bishopsgate Suite) }\end{array}$ \\
\hline $\begin{array}{l}410 \text { - Day Ahead Hourly Electricity Price } \\
\text { Prediction in ISO New England Market using } \\
\text { Neuro-Fuzzy Systems (Presenter: Vassilis } \\
\text { Kodogiannis, United Kingdom) } \\
328 \text { - An Overview of On-Chip Cache } \\
\text { Coherence Protocols (Presenter: Zainab Alwaisi, } \\
\text { United Kingdom) } \\
119 \text { - A TT\&C Management Method for } \\
\text { Satellite Constellation Networking Process } \\
\text { (Presenter: Jing Li, China) } \\
194 \text { - iSAFETY - Integrated System for an } \\
\text { Automatic Support to Fishing Vessel Security } \\
\text { (Presenter: Salvatore Aronica, Italy) } \\
134 \text { - A Review of Technician and Task } \\
\text { Scheduling Problems, Datasets and Solution } \\
\text { Approaches (Presenter: Amy Khalfay, United } \\
\text { Kingdom) } \\
175 \text { - Reasoning and Algorithm Selection } \\
\text { Augmented Symbolic Segmentation (Presenter: } \\
\text { Martin Lukac, Kazakhstan) } \\
405 \text { - A Model and Prototype of a Proactive } \\
\text { Multi-Type Context-Aware Recommender } \\
\text { System (Presenter: Abdallatif Abuissa, } \\
\text { Palestine) } \\
287 \text { - Recommending Highlights in Anime } \\
\text { Movies: Mining the Real-Time User Comments } \\
\text { "Danmaku" (Presenter: Yifan He, China) }\end{array}$ & $\begin{array}{l}292 \text { - Financial Discussion Boards Irregularities } \\
\text { Detection System (FDBs-IDS) using Information } \\
\text { Extraction (Presenter: Majdi Owda, United } \\
\text { Kingdom) } \\
386 \text { - A Heuristic Attack Detection Approach } \\
\text { using the "Least Weighted" Attributes for Cyber } \\
\text { Security Data (Presenter: Kato Mivule, United } \\
\text { States) } \\
256 \text { - Phased Array Antenna for the Application } \\
\text { of Device Free Localization in Indoor } \\
\text { Environments (Presenter: Ata ur Rehman, } \\
\text { Pakistan) } \\
198 \text { - Re-Evaluation of Combined Markov- } \\
\text { Bayes Models for Host Intrusion Detection on } \\
\text { the ADFA Dataset (Presenter: Tajjeeddine } \\
\text { Rachidi, Morocco) } \\
132 \text { - Hand Signature and Handwriting } \\
\text { Recognition as Identification of the Writer } \\
\text { using Gray Level Co-Occurrence Matrix and } \\
\text { Bootstrap (Presenter: Teny Handhayani, } \\
\text { Indonesia) } \\
80 \text { - Development of Intelligent Tools for } \\
\text { Recognizing Cockroaches in the Forensic } \\
\text { Entomology Context (Presenter: Eduardo } \\
\text { Vázquez-Santacruz, Mexico) }\end{array}$ & $\begin{array}{l}42 \text { - Detection By Registration (Presenter: } \\
\text { Michael Greenspan, Canada) } \\
316 \text { - Crack Detection in Concrete Elements } \\
\text { from RGB Pictures using Modified Line } \\
\text { Detection Kernels (Presenter: Luis Sanchez, } \\
\text { Spain) } \\
109 \text { - Adaptive Signal Processing Strategy for a } \\
\text { Wind Farm System Fault Accommodation } \\
\text { (Presenter: Silvio Simani, Italy) } \\
165 \text { - Automated Representation of Non- } \\
\text { Emotional Expressivity to Facilitate } \\
\text { Understanding of Facial Mobility: Preliminary } \\
\text { Findings (Presenter: Kathy Clawson, United } \\
\text { Kingdom) } \\
289 \text { - Temporal Segmentation of Human } \\
\text { Actions in Video Sequences (Presenter: Juan } \\
\text { Climent, Spain) } \\
349 \text { - Unsupervised Image Segmentation using } \\
\text { Lab Color Space (Presenter: Junaid Baber, } \\
\text { Pakistan) } \\
164 \text { - Microrecording of Writer's Cramp Signals } \\
\text { with Indigenously Developed Advanced Real- } \\
\text { Time Multi-Channel Intelligent-EMG-System } \\
\text { (Presenter: Venkateshwarla Rama Raju, India) }\end{array}$ & $\begin{array}{l}56 \text { - CAMABOT, An Assistance Device for } \\
\text { Mexican Society with Motor Disabilities } \\
\text { (Presenter: Eduardo Vázquez-Santacruz, } \\
\text { Mexico) } \\
33 \text { - A Novel Approach to Register Sonar Data } \\
\text { for Underwater Robot Localization (Presenter: } \\
\text { Antoni Burguera, Spain) } \\
73 \text { - Using Confidence Factors to Share Control } \\
\text { between a Mobile Robot Tele-Operater and } \\
\text { Ultrasonic Sensors (Presenter: Nils Bausch, } \\
\text { United Kingdom) } \\
273 \text { - Design and Development of a Skinny } \\
\text { Bidirectional Soft Glove for Post-Stroke Hand } \\
\text { Rehabilitation (Presenter: Boran Wang, New } \\
\text { Zealand) } \\
393 \text { - Practical Formation Control of Swarm } \\
\text { Robots Using Mobile Agents (Presenter: } \\
\text { Hideaki Yajima, Japan) } \\
312 \text { - Real-World Large-Step Stair-Climbing for } \\
\text { Small Humanoids using Evolutionary } \\
\text { Computation (Presenter: Nikolaos Mavridis, } \\
\text { Russia) } \\
278 \text { - Sensor Fusion System for Autonomous } \\
\text { Localization of Mobile Robots (Presenter: } \\
\text { Manuel Olivares Avila, Chile) }\end{array}$ \\
\hline
\end{tabular}


8th September (3:45 pm - 5:15 pm)

PAPER PRESENTATIONS

\begin{tabular}{|c|c|c|c|}
\hline $\begin{array}{l}\text { Session 21: Ambient Intelligence } \\
\text { Session Chair: Amanda Peart } \\
\text { (Ludgate Suite) }\end{array}$ & $\begin{array}{l}\text { Session 22: Neural Networks } \\
\text { Session Chair: Kohei Arai } \\
\text { (Walbrook Suite) }\end{array}$ & $\begin{array}{l}\text { Session 23: Natural Language Processing } \\
\text { Session Chair: Yaxin } \mathrm{Bi} \\
\text { (Fleet Suite) }\end{array}$ & $\begin{array}{l}\text { Session 24: Robotics } \\
\text { Session Chair: Vassilis S. Kodogiannis } \\
\text { (Bishopsgate Suite) }\end{array}$ \\
\hline $\begin{array}{l}179 \text { - Drivers, Standards and Platforms for the } \\
\text { IoT: Towards a Digital VICINITY (Presenter: } \\
\text { Carna Radojicic, Germany) } \\
300 \text { - IoT Enabled Smart Buildings: A } \\
\text { Systematic Review (Presenter: Muhammad } \\
\text { Rizwan Bashir, Australia) } \\
374 \text { - Smart City Project using Proximity } \\
\text { Marketing Technology (Presenter: Lamya } \\
\text { Albraheem, Saudi Arabia) } \\
258 \text { - A New Technique for Saving Water } \\
\text { Trapped Inside Home Water Networks } \\
\text { (Presenter: Jihad AlSadah, Saudi Arabia) } \\
163 \text { - A Novel Design and Miniaturization of a } \\
\text { Scalp Implantable Circular Patch Antenna at } \\
\text { ISM Band for Biomedical Application } \\
\text { (Presenter: Raja Rashidul Hasan, Bangladesh) } \\
76 \text { - Restoring the Chinese Calendar Li Fa and } \\
\text { the Cosmic Breath Qi to the Real World } \\
\text { (Presenter: Rey Tiquia, Australia) }\end{array}$ & $\begin{array}{l}177 \text { - Hybrid Functional Networks for PVT } \\
\text { Characterisation (Presenter: Munirudeen Oloso, } \\
\text { United Kingdom) } \\
304 \text { - Cryptocurrency Portfolio Management } \\
\text { with Deep Reinforcement Learning (Presenter: } \\
\text { Zhengyao Jiang, Jinjun Liang, China) } \\
305 \text { - Survey on a Neural Network for Non } \\
\text { Linear Estimation of Aerodynamic Angles } \\
\text { (Presenter: Alberto Brandl, Italy) } \\
169 \text { - On Non-Iterative Training of a Neural } \\
\text { Classifier Part-II: Clustering of Points and Their } \\
\text { Classification using an NN Architecture } \\
\text { (Presenter: Damodhar Rao Kandula, India) } \\
168 \text { - On Non-Iterative Training of a Neural } \\
\text { Classifier Part-I: Separation of Points by Planes } \\
\text { (Presenter: Kumar Eswaran, India) }\end{array}$ & $\begin{array}{l}201 \text { - Comparison of Semantic Vectors with } \\
\text { Reduced Precision using the Cosine Similarity } \\
\text { Measure (Presenter: Michał Karwatowski, } \\
\text { Poland) } \\
365 \text { - Content Analysis of MooC Forums } \\
\text { (Presenter: Anat Cohen, Israel) } \\
26 \text { - Improving Arabic Document Clustering } \\
\text { using K-Means Algorithm and Particle Swarm } \\
\text { Optimization (Presenter: Abdullah Daoud, } \\
\text { Egypt) } \\
255 \text { - Spelling Correction in English: Joint Use } \\
\text { of Bi-Grams and Chunking (Presenter: Vladimir } \\
\text { Polyakov, Russian Federation) } \\
383 \text { - Question Answer System for Online } \\
\text { Feedable New Born Chatbot (Presenter: } \\
\text { Sameera Abdul-Kader, United Kingdom) } \\
228 \text { - An Efficient Rank Based Arabic Root } \\
\text { Extractor (Presenter: Nahla Belal, Egypt) }\end{array}$ & $\begin{array}{l}306 \text { - A Compact, Low-Cost Unmanned Surface } \\
\text { Vehicle for Shallow Inshore Applications } \\
\text { (Presenter: Zuhayr Rymansaib, Benjamin } \\
\text { Metcalfe, United Kingdom) } \\
29 \text { - A Comparative Study of the Optimal } \\
\text { Control Design using Evolutionary Algorithms: } \\
\text { Application on a Close-loop System (Presenter: } \\
\text { Alireza Mohseni, Canada) } \\
379 \text { - The Effect of Mono and Biarticular } \\
\text { Muscles on the Dynamic of Walking Bipedal } \\
\text { Robot (Presenter: Brahim Fernini, Algeria) } \\
296 \text { - Mobile Robot Localization Via Sensor } \\
\text { Fusion Algorithms (Presenter: Turker Erguzel, } \\
\text { Turkey) } \\
301 \text { - Development of a Control System for } \\
\text { Electric Wheelchairs based on Head } \\
\text { Movements (Presenter: Guilherme Maciel, } \\
\text { Brazil) } \\
49 \text { - Robustness of Cooperative Behaviour } \\
\text { Model on N Robot-based Multi-Robot Systems: } \\
\text { Application to Mine Emergency and Disaster } \\
\text { Management (Presenter: Isaac Osunmakinde, } \\
\text { Chika Yinka-Banjo) }\end{array}$ \\
\hline
\end{tabular}

